\title{
Earlier treatment improves the chances of complete relief from postherpetic neuralgia.
}

\author{
Hee Young Kim ${ }^{1}$,Young-Min Ok ${ }^{1}$, Ji-Seok Baik², Tae-Kyun Kim ${ }^{1}$,Sang-Wook Shin', Hae-Kyu Kim², Kyung-Hoon Kim ${ }^{1}$ \\ 1 Department of Anesthesia and Pain Medicine, Pusan National University Yangsan Hospital, Yangsan, Korea \\ 2 Department of Anesthesia and Pain Medicine, Pusan National University Hospital, Busan, Korea
}

\section{Introduction}

Postherpetic neuralgia (PHN) is usually defined as pain persisting for more than 120 days after the onset of herpes zoster rash.

- Among PHN patients who commonly visit a pain physician rather than a dermatologist, complete relief from pain in response to active treatment can sometimes be observed in the clinical field.

This study evaluated the incidence of complete relief from PHN and the factors influencing it.

\section{Methods}

A retrospective chart review was performed on patients with PHN at a university-based pain clinic over 7 years.

The responders were defined as patients who had complete relief from pain after 1 year of active treatment.

- Age, sex, duration of PHN at their first visit, and dermatomal distribution were compared in the responder and non-responder groups.

- Responders were also compared according to these factors.

\section{Results}

\begin{tabular}{|c|c|c|c|c|c|c|c|c|c|c|c|c|c|c|c|c|}
\hline & \multicolumn{8}{|c|}{ Number of Responders $=35(29.9 \%)$} & \multicolumn{8}{|c|}{ Number of Non-Responders $=82(70.1 \%)$} \\
\hline Age (y) & $<50$ & \multicolumn{3}{|c|}{$50-59$} & \multicolumn{2}{|l|}{$60-69$} & \multicolumn{2}{|l|}{$\geq 70$} & $<50$ & \multicolumn{2}{|c|}{$50-59$} & \multicolumn{3}{|c|}{$60-69$} & \multicolumn{2}{|l|}{$\geq 70$} \\
\hline Number $(\mathrm{N})$ & 2 & \multicolumn{3}{|c|}{10} & \multicolumn{2}{|l|}{9} & \multicolumn{2}{|l|}{14} & 7 & \multicolumn{2}{|l|}{14} & \multicolumn{3}{|l|}{24} & \multicolumn{2}{|l|}{37} \\
\hline Sex & \multicolumn{4}{|l|}{ M } & \multicolumn{4}{|l|}{$\mathrm{F}$} & \multicolumn{3}{|l|}{ M } & \multicolumn{5}{|l|}{$\mathrm{F}$} \\
\hline $\mathrm{N}$ & \multicolumn{4}{|l|}{11} & \multicolumn{4}{|l|}{24} & \multicolumn{3}{|l|}{37} & \multicolumn{5}{|l|}{45} \\
\hline $\begin{array}{l}\text { Duration of } \mathrm{PHN} \\
\text { at their first visit }\end{array}$ & $\begin{array}{l}<6 \\
\text { (month) } \\
\end{array}$ & \multicolumn{2}{|c|}{$6-9$} & $9-12$ & $12-15$ & $15-18$ & $18-24$ & $24 \geq$ & $<6$ & $6-9$ & $9-12$ & \multicolumn{2}{|c|}{$12-15$} & $15-18$ & $18-24$ & $24 \geq$ \\
\hline$N$ & 20 & \multicolumn{2}{|l|}{5} & 3 & 2 & 2 & 1 & 2 & 38 & 12 & 4 & 6 & & 2 & 9 & 11 \\
\hline Diabetes & \multicolumn{4}{|l|}{-} & \multicolumn{4}{|l|}{+} & \multicolumn{4}{|l|}{-} & \multicolumn{4}{|c|}{+} \\
\hline $\mathrm{N}$ & \multicolumn{4}{|l|}{33} & \multicolumn{4}{|l|}{2} & \multicolumn{4}{|l|}{80} & 2 & & & \\
\hline $\begin{array}{l}\text { Cancer with } \\
\text { immunosuppression }\end{array}$ & - & & & & + & & & & - & & & & + & & & \\
\hline$N$ & 34 & & & & 1 & & & & 80 & & & & 2 & & & \\
\hline $\begin{array}{l}\text { Dermatomal } \\
\text { distribution }\end{array}$ & $C N \vee(V$ & & Cer & ical (C) & Thoracic & $(\mathrm{T})$ & Lumbosac & $\mathrm{al}(\mathrm{L})$ & V & & C & & $\mathrm{T}$ & & L & \\
\hline$N$ & 9 & & 4 & & 18 & & 4 & & 17 & & 13 & & 43 & & 9 & \\
\hline
\end{tabular}

Table 1. Comparison of age, sex, duration of PHN, underlying disease, and dermatomal distribution

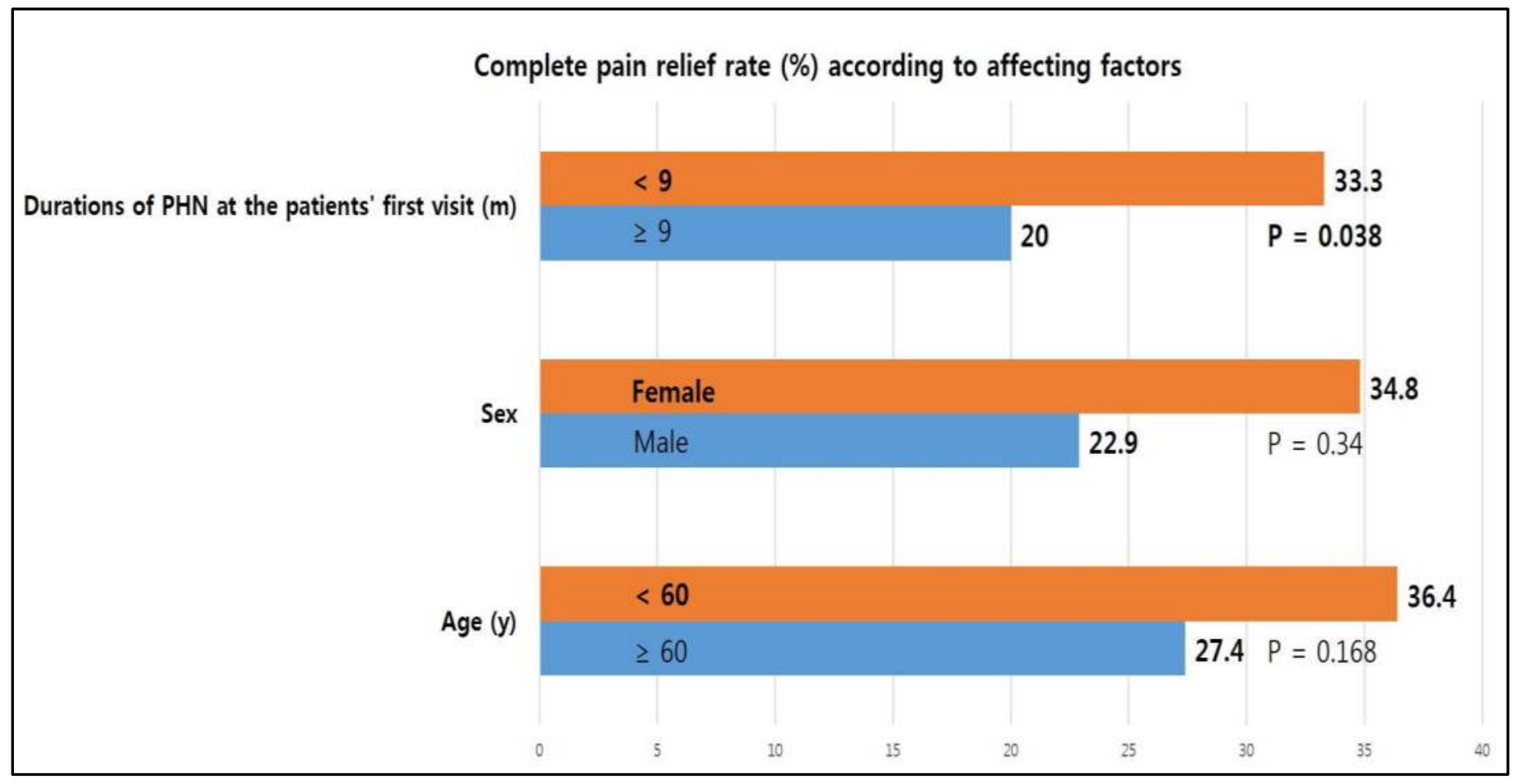

\section{Conclusions}

Fig 1. Complete pain relief rate (\%) according to affecting factors

Almost 30\% of PHN patients received complete pain relief.

- Those who sought treatment earlier, within 9 months, in a pain clinic, received a better result. 